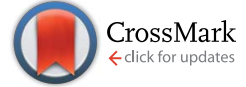

Cite this: RSC Adv., 2016, 6, 69117

Received 27th May 2016 Accepted 14th July 2016

DOI: 10.1039/c6ra13801b

www.rsc.org/advances

\title{
Tunable surface topography in fluoropolymers using photo-embossing $\dagger$
}

\begin{abstract}
S. Kommeren, ${ }^{* a}$ T. Sullivan ${ }^{a}$ and C. W. M. Bastiaansen ${ }^{a b}$
Novel methods that allow creation and tunable control of surface relief in polymer films are of key interest in the search for novel low surface energy materials. For example, photochemically cross-linked (UV-cured) high-performance fluoropolymer films with an engineered surface relief of precisely defined shapes and dimensions could have widespread applications. Here, we report a fabrication method based on photo-embossing that provides the ability to create surface relief in fluorinated elastomers. The height and shape of the surface relief structures can be altered as desired by changing the processing conditions such as energy dose, monomer composition and the added solvent volumes. Surface relief structures with heights of up to $9 \mu \mathrm{m}$ have been obtained using a photomask with a $40 \mu \mathrm{m}$ pitch. We demonstrate that surface relief structures with a broad range of shapes and dimensions can be created if appropriate photomasks are available.
\end{abstract}

\subsection{Introduction}

The ability to easily generate surface relief in a controlled manner in films, coatings and/or fibers based on fluoropolymers with a low surface energy is highly desirable from a commercial viewpoint and has many practical applications. ${ }^{1}$ Manufactured material surfaces are rarely smooth at the micro or nanoscale and often incorporate random or non-random surface relief structures and shapes from either the manufacturing process or during subsequent application. ${ }^{2}$ However, the ability to precisely control surface relief structures is key to many industrial processes, for example in silicon chip manufacture, in drag-reducing surfaces, superhydrophobic or superoleophobic surfaces or on smart switchable materials for various applications. ${ }^{3-7}$ Thus novel, cheap and facile methods of generating tuneable surface relief structures are of continued widespread interest. ${ }^{6}$ Control of surface relief structures is also of importance in biotechnology applications, where control of surface roughness at the micro and nanoscale can have important implications in the production and performance of technologies such as body implants and prostheses, in dental work and surgical devices. ${ }^{\mathbf{8} 9}$ Bacterial or mammalian cell behaviour has been shown to be affected at interfaces by surface

${ }^{a}$ Department of Chemical Engineering and Chemistry, Functional Organic Materials \& Devices (SFD), De Rondom 70 - 5612 AP, Eindhoven, The Netherlands. E-mail: s. kommeren@tue.nl

${ }^{b}$ School of Engineering and Materials Science, Queen Mary University of London, Mile End Road, London E1 4NS, UK

$\dagger$ Electronic supplementary information (ESI) available: The mechanical properties of different fluoropolymers are shown and further Raman spectroscopy data about the composition of the fluoropolymer films are included. See DOI: 10.1039/c6ra13801b relief structures, and it currently appears that cell removal, mobility and survival are all influenced by the shape and nature of the surface relief structures present. ${ }^{\mathbf{1 0}}$

Fluoropolymers are of general interest for applications such as antifouling due to their stability, low surface energy and their suitability for application as coatings. ${ }^{11,12}$ Omniphobic surfaces (surfaces with the capability to repel various simple and complex liquids e.g. water, hydrocarbons, crude oil or blood) can be also produced using micro-patterned crosslinked fluorogels containing perfluorinated oils such as Fluorinert FC-70. ${ }^{11,13}$

The ability to tune both the bulk material and surface properties for precise control over the physical properties and corresponding behaviour of the material has important advantages for a variety of applications. ${ }^{11,14}$ Fluoropolymers that range from opaque to transparent at visible wavelengths, and with mechanical properties extending from a stiff, rigid plastic to a soft elastomer, have been reported. ${ }^{11}$

Many methods currently utilised for creation of controlled surface relief require one or more development and/or chemical etching steps, or are made via contact embossing or mould replication. ${ }^{15-17}$ The use of a mould often limits the height of the surface relief to predefined dimensions. Here, we demonstrate that photo-embossing, a convenient and economic process to form complex surface relief in polymer thin films, provides a convenient, reproducible and tunable method of creating surface relief in fluorinated polymers. We demonstrate that creation of well-defined surface relief structures is possible, and that the aspect ratio (height/width) and feature height can be tuned by altering the pitch (the width of one period) of the photomask grating, energy dose, monomer composition, and the addition of volatile solvents. 
Formation of surface relief during the photopolymerization and the UV-curing process as described here for a fluorinated (meth)acrylate system has a number of advantages. When compared to thermally induced polymerization for example (i) the polymer formation is relatively fast, (ii) it is carried out at room temperature, (iii) the initiation permits local polymerization as it only occurs in the illuminated areas, (iv) the method does not require chemical etching and (v) it has the potential to be scaled-up to produce surface relief over larger surface areas.

Photo-embossing using UV photopolymerization, as a means of producing patterned polymers for use in optical and display applications, has proven to be a versatile and facile means of patterning materials. The polymerization reaction is only initiated in areas exposed to UV-light and the subsequent change in chemical potential provides a driving force for the monomers in the unexposed areas to diffuse to the exposed areas. ${ }^{18,19}$ Differences in UV-light intensity, energy dosage, temperature, grating pitch, monomer size and reactive groups, inhibitors, RAFT agents, polymer binder and monomer/polymer ratios can be tuned to produce different surface relief structures. ${ }^{\mathbf{2 0}-27}$

In the system described here, diffusion of the monomers to the exposed regions of the sample results in an increase in height of the polymer in these regions. Previous work by Leewis et al., provided a comprehensive model of the mechanism of photo-embossing based upon the Flory-Huggins model of thermodynamic interactions within monomer-monomer and monomer-polymer solutions, showing the influences of monomer reactivity, concentration gradients, cross-linking ability, size, shape and monomer-polymer interaction effects for different mono- and di(meth)acrylate monomer systems. ${ }^{\mathbf{1 8 , 1 9}}$ The model thus developed allows prediction of monomer migration patterns within different systems and ultimate selection of different monomers and conditions for the final application (optical gratings, optical diffusers, relief structures etc.). ${ }^{18,19}$ The subsequent work of Sánchez et al., further facilitated the application of photo-embossing by systematic study of the effects of pattern dimensions, energy dose, development temperature, film thickness, and photopolymer blend composition, allowing selection of optimum conditions to create the desired surface relief structures. ${ }^{20,21}$ Greater aspect ratios were achieved by Hermans et al. and Perelaer et al. through the addition of reversible addition-fragmentation chain transfer (RAFT) agents and later by Hughes-Brittain et al. by varying the polymer binder of the photopolymer blend. ${ }^{22-25}$

In contrast to the process described here, the photopolymer mixtures used for 'classical' photo-embossing were designed so that the photopolymer mixtures are below the glass transition temperature $\left(T_{\mathrm{g}}\right)$ at room temperature..$^{\mathbf{2 0 - 2 4}}$ Radicals are generated in the exposed areas during exposure to UV-light with the advantage that contact photomask exposure can be performed such that different exposures can be super-imposed to generate, for instance, hierarchical surface relief structures. However, due to the lack of mobility in the glassy photopolymer monomer, diffusion to the reactive sites is restricted and polymerization is minimal because the free radicals are captured in an immobile glassy matrix. A subsequent heating step increases the mobility in the photopolymer, which allows monomers to polymerize in the exposed areas and diffusion to those areas takes place. ${ }^{\mathbf{2 0 - 2 6}}$

In contrast to previous studies, fluorinated monomers were utilised here to further broaden the range of properties and applications for photo-embossing. The system described here consists of a two liquid monomers: a monoacrylate (PFDA) and a dimethacrylate crosslinker (PFPE-DMA). These monomers, used in combination with a fluorinated lubricant, have recently been reported to create omniphobic materials that have subsequently shown both shape memory behaviour and excellent anti-adhesion properties for a range of protein, blood and mammalian cell assays. ${ }^{11}$

\subsection{Materials and methods}

A monoacrylate, $1 H, 1 H, 2 H, 2 H$-perfluorodecyl acrylate (PFDA), was purchased from Sigma-Aldrich and a crosslinker, perfluoropolyether dimethacrylate (PFPE-DMA, Fomblin MD40), was provided by Solvay Specialty Polymers. The molecular structures of the two fluorinated monomers are shown in Fig. 1. A photoinitiator, Irgacure 819, was purchased from BASF and dichloromethane (DCM) was obtained from Biosolve. All materials were used without further modification.

PFDA was mixed with PFPE-DMA in different ratios to yield fluorinated polymer films. It was envisioned that the morphology of the fluorogel polymer network could be precisely tuned from semi-crystalline to amorphous by specifying the identity and/or amount of monomer in the matrix, as was shown by Yao et al. ${ }^{11}$ The photoinitiator is dissolved in DCM, since it is not soluble in the monomers, in the desired concentration and subsequently added to the monomer mixture. The standard mixture has 87 vol\% of the monomer mixture (1:1 volume ratio of PFDA and PFPE-DMA) and $13 \mathrm{vol} \%$ of DCM in which $9 \mathrm{mg} \mathrm{mL} \mathrm{mL}^{-1}$ of photoinitiator is dissolved. For a $1 \mathrm{~mL}$ monomer mixture this results in $1.17 \mathrm{mg}$ of photoinitiator. The naming convention is monomer-crosslinker-vol\% monomer e.g.: PFDA-MD40-50.

\section{Substrate preparation}

Glass substrates $(3 \times 3 \mathrm{~cm})$ were cleaned by means of sonication (in acetone, $15 \mathrm{~min}$ ) followed by treatment in an UV-ozone photoreactor (Ultra Violet Products, PR-100, 20 min). The surface of the glass substrates were modified by spin-coating a 3-(trimethoxysilyl) propyl methacrylate solution (1\% v/v solution in a $1: 1$ water-isopropanol mixture) on the activated glass substrate for $30 \mathrm{~s}$ at $3000 \mathrm{rpm}$. After curing for $10 \mathrm{~min}$ at $110^{\circ} \mathrm{C}$, the substrates were ready for use.

Substrate-attached photo-embossed films were prepared in home-made cells (see Fig. 2) consisting of a lower silane methacrylate functionalized glass slide glued to an upper sodalime glass chromium oxide photomask. The cell is glued together at the edges with 2 strips of double sided tape (Tesa, photostrip) of 2-3 $\mathrm{mm}$ wide at the edges. A range of photomasks with different feature shapes, dimensions and spacing were utilised to determine effects of the grating pitch on obtained feature shapes and heights. The cells were capillary-filled with 
a)<smiles>C=CC(=O)OCCC(C)(C)C(F)(F)F</smiles>

b)

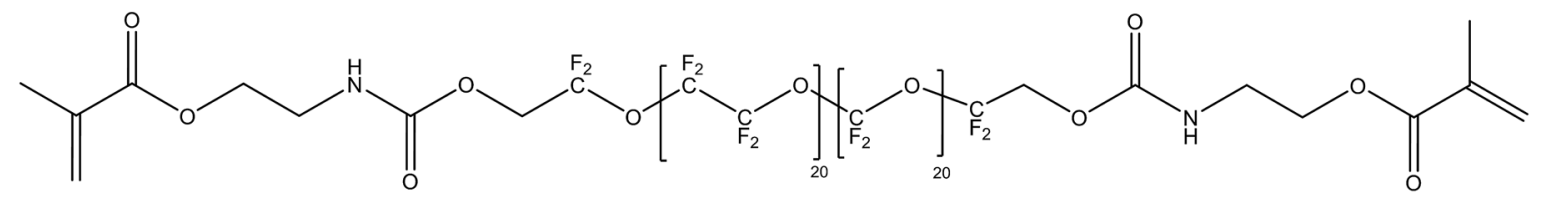

Fig. 1 The molecular structure of both monomers is shown above. (a) Perfluorodecyl acrylate and (b) perfluoropolyether dimethacrylate. The ratio of these two monomers can be tuned to produce different mechanical and optical materials properties.

the monomer-crosslinker mixture and subsequently exposed to UV light (EXFO Omnicure S2000 lamp) with an intensity of $5 \mathrm{~mW} \mathrm{~cm}{ }^{-2}$ in the UVA range $(320-390 \mathrm{~nm})$. Cells were subsequently turned over for a flood exposure for 128 seconds at $5 \mathrm{~mW} \mathrm{~cm}{ }^{-2}$ to ensure complete polymerization of the monomer mixture.

\section{Surface structure characterisation}

The thickness of the resulting films and dimensions of the resulting surface relief structures were measured using white light interferometry (Fogale Nanotech Zoomsurf 3D). Error bars indicate the standard deviation over a minimum of 6 measurements distributed over 2 separate samples. It has to be noted that for the majority of the surface relief the slope was too steep to be measured by white light interferometry. Height profiles that are shown have an interpolated slope. Scanning electron microscopy (Jeol JSM-5600) was therefore used to confirm the shape of the surface relief. Samples were prepared for SEM by adhering films supported with a glass substrate to a)

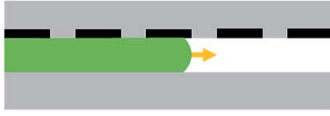

b)
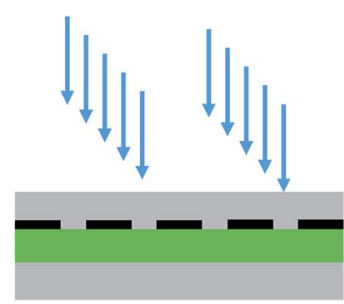

c)

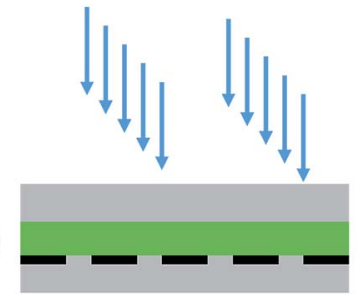

d)

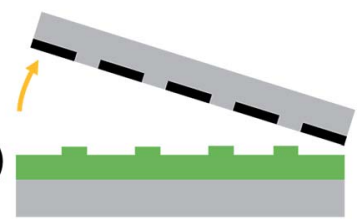

Fig. 2 Schematic representation of the method for photo-embossing liquid monomers. (a) The cell is capillary filled with the liquid monomer mixture. (b) The cell is exposed via the photomask and (c) subsequently a flood exposure is done via the substrate to polymerize the remaining monomers. (d) The photomask can then be removed by opening the cell, leaving a fluoropolymer with the desired surface relief on the substrate.
$15 \mathrm{~mm}$ Al stubs with carbon tape and subsequent sputtercoated with Au (approximately $15 \mathrm{~nm}$ ) in an argon atmosphere. Confocal Raman spectroscopy measurements (Horiba Raman fibre microscope Olympus BX40) were performed to look at the compositional differences between areas exposed and unexposed through the photomask. The Raman spectroscopy measurements were done in a closed cell with an Olympus UPlanApo $100 \times / 1,35$ oil immersion objective.

\subsection{Results and discussion}

The monomer ratio can be adjusted to create a wide range of mechanical and optical properties; from a soft elastomer to a stiff rigid plastic with a modulus of one order of magnitude higher and from transparent to opaque. ${ }^{11}$ The mechanical properties of the films are shown in the ESI in Fig. S1. $\dagger$

The system described here uses a cell configuration as shown in Fig. 2, where the cell is capillary filled with the liquid monomer mixture. In contrast to 'classical' photo-embossing the mobility of the monomers is high and polymerization starts immediately upon exposure to UV-light. Monomers are immediately able to diffuse to the exposed areas. The (3D) height profile of a photo-embossed film with a $40 \mu \mathrm{m}$ pitch is shown in Fig. 3. The height of the obtained surface relief structures is dependent on the energy dose given by the UV-light in the photomask exposure step. By increasing the energy dose, more monomers are polymerized in the exposed areas and therefore more monomers diffuse from the unexposed areas to the exposed areas, creating a (higher) surface structure. It has been shown that in this system the height of the surface relief structures increases with increasing energy dose (Fig. 3a). The plateau that is reached after $200-250 \mathrm{~mJ} \mathrm{~cm}^{-2}$ indicates that the gelation or vitrification point is reached and further diffusion does not occur. In Fig. 3a the dependence of height on energy dose is shown for several line patterns ranging from a pitch of $40 \mu \mathrm{m}$ to $5 \mu \mathrm{m}$ (with a transparency ratio of 0.5 ). Larger pitches result in higher surface relief structures, since more material is able to diffuse to the exposed areas.

The effect of the monomer mixture composition on the height of the generated surface relief structures was also investigated. Only the monomer ratio was varied and all other variables were kept constant. From Fig. 4 it can be seen that the height of the surface relief structures is dependent on the 

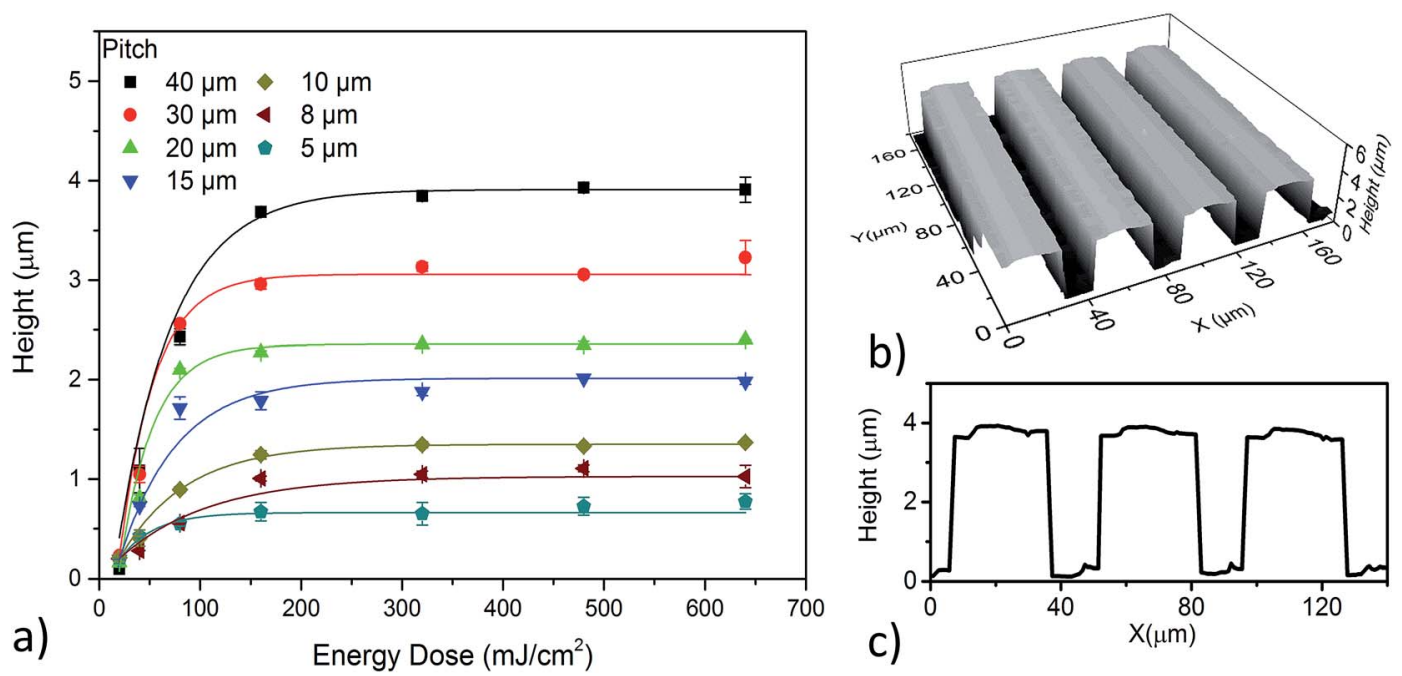

Fig. 3 (a) The height of the surface relief structures increases with increasing energy dose, until a plateau is reached after $200-250 \mathrm{~mJ} \mathrm{~cm}^{-2}$. The effect of the pitch of the photomask grating can also be seen, where increasing the pitch results in higher surface relief structures. An example of a typical 3D (b) and a 2D height profile (c) of structures generated using a photomask with a $40 \mu \mathrm{m}$ pitch and an energy dose of $640 \mathrm{~mJ} \mathrm{~cm}^{-2}$ is shown.

composition of the monomer mixture. Since the PFPE-DMA has a relatively high molecular weight $\left(4200 \mathrm{~g} \mathrm{~mol}^{-1}\right)$ and the PFDA a comparatively low molecular weight $\left(518 \mathrm{~g} \mathrm{~mol}^{-1}\right)$, viscosity differs with the monomer mixture ratios. Upon increasing the amount of PFDA the monomer mixture becomes less viscous and therefore the monomers will have more mobility and readily diffuse to the exposed areas. In short, an increase in PFDA content leads to higher surface relief structures. However, this does not hold for the highest amount of PFDA (95 vol\%) and this is likely due to the polymer film becoming semicrystalline and opaque during polymerization at high volume percentages of PFDA, in agreement with the results reported by

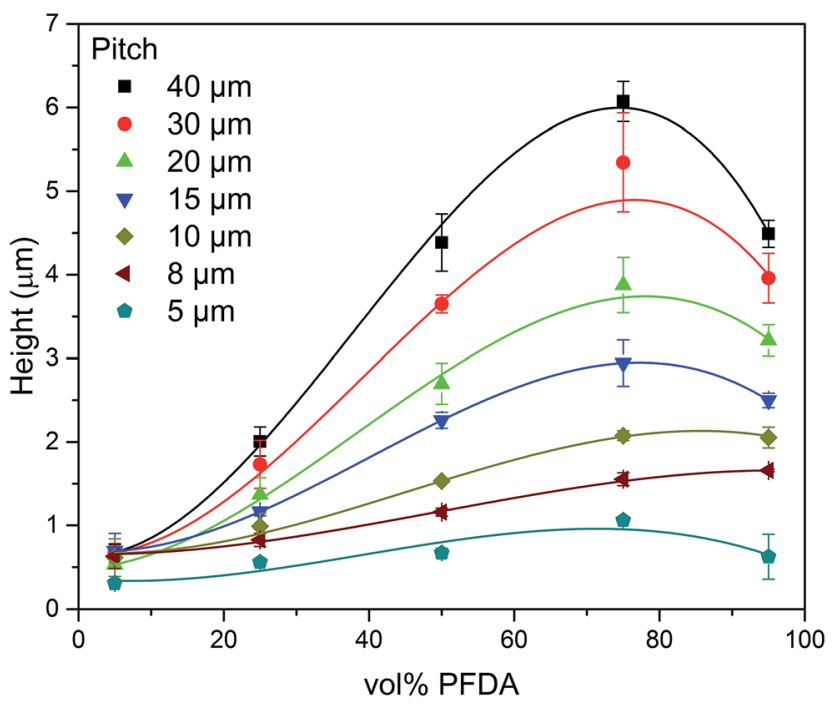

Fig. 4 Increasing the volume percentage (up to 75 vol\%) of PFDA decreases the viscosity resulting in generally higher surface relief structures. However, at $>95$ vol\% PFDA the height decreases due to the change in optical properties of the film during polymerization.
Yao et al. ${ }^{11}$ This semi-crystallinity leads to light scattering in the unexposed areas, resulting in lower surface relief structures.

In the results presented above, DCM (13 vol\%), was used to dissolve the photoinitiator in the monomer mixtures. This procedure was adopted because the pure photoinitiator was insoluble in the monomers. The added volume of DCM was varied to study the effect of an added solvent to the mixture and the resultant surface relief generated. Fig. 5a shows that the volume percentage of DCM added to the monomer mixture influences the final height of the surface relief structures. The observed effects are due to the increase in mobility of the monomers with increased vol\% of added solvent.

Such counter diffusion combined with enhanced mobility of the monomers enables the creation of surface relief structures up to $9 \mu \mathrm{m}$ in height given a photomask pitch of $40 \mu \mathrm{m}$. Upon opening the cell, the DCM will evaporate and only the photoembossed fluoropolymer film remains. A SEM image of such a photo-embossed surface relief structure is shown in Fig. 5b.

The thickness of the polymerised film was also measured for increasing volumes of added solvent, showing a decrease in final film thickness with increasing volumes of added solvent (Fig. S2 $\uparrow$ ). The initial film thickness is determined by cell spacers which are approximately $100 \mu \mathrm{m}$ in thickness and the difference in initial and final film thickness approximately corresponds to the vol\% of added solvent.

Confocal Raman spectroscopy was used to determine the local composition of the fluoropolymer. In Fig. S3 $\uparrow$ the Raman spectra of PFDA and of MD40 are shown. For PFDA the characteristic peak at $725 \mathrm{~cm}^{-1}$ from the $\mathrm{CF}_{3}$ vibration is used to determine the presence of the monomer. For MD40 the characteristic $\mathrm{C}-\mathrm{O}-\mathrm{C}$ peak at $825 \mathrm{~cm}^{-1}$ is used to determine the presence of the crosslinker.

The composition of the fluoropolymer film is scanned in approximately the middle of the sample and the scanning 

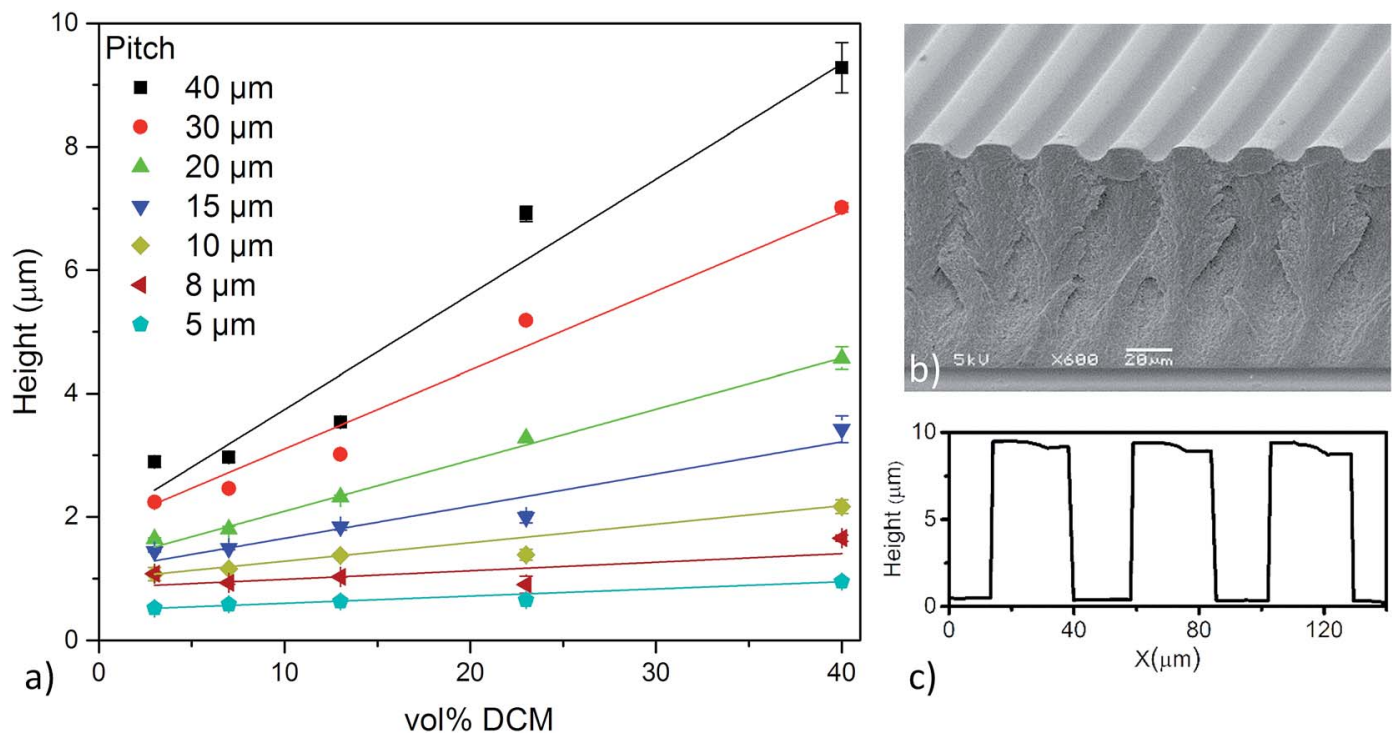

Fig. 5 (a) The height of the surface relief structures can be increased by increasing the amount of DCM in the photo-embossing mixture. It is likely that the increase in solvent increases the mobility of the reactive monomers and the DCM diffuses to the unexposed areas. (b) A SEM image of PFDA-MD40-50 with 40 vol\% DCM at a $40 \mu \mathrm{m}$ pitch. The surface relief structures are approximately $9 \mu \mathrm{m}$ high, as can be seen in the interferometer height profile (c). The final thickness of the fluoropolymer film is approximately $70 \mu \mathrm{m}$.

direction is perpendicular to the photo-embossed line pattern. In Fig. 6a the Raman spectra are shown versus the measurement position with respect to areas exposed and unexposed to UV light through the photomask. Four periods of exposed/ unexposed areas were measured as can be seen in the schematic of Fig. 6b. The $\mathrm{CF}_{3}$ peak (at $725 \mathrm{~cm}^{-1}$ ) of PFDA has a higher intensity in areas exposed during the photoembossing process. The opposite can be seen for the intensity of the C-O-C peak (at $825 \mathrm{~cm}^{-1}$ ) of the MD40, which has a clear intensity peak in the unexposed areas. This indicates that the PFDA diffuses to the exposed areas. Similar to the height profiles, it appears that the monomer diffusion of the PFDA towards the exposed areas leads to a broadening of the surface relief structures, compared to the original photomask dimensions. This can be also seen in Fig. 6 a where the $\mathrm{CF}_{3}$ peak is broader then the photomask dimension of $20 \mu \mathrm{m}$.

The composition of the fluoropolymer was also investigated throughout the thickness of the film. In Fig. S4† it is shown that
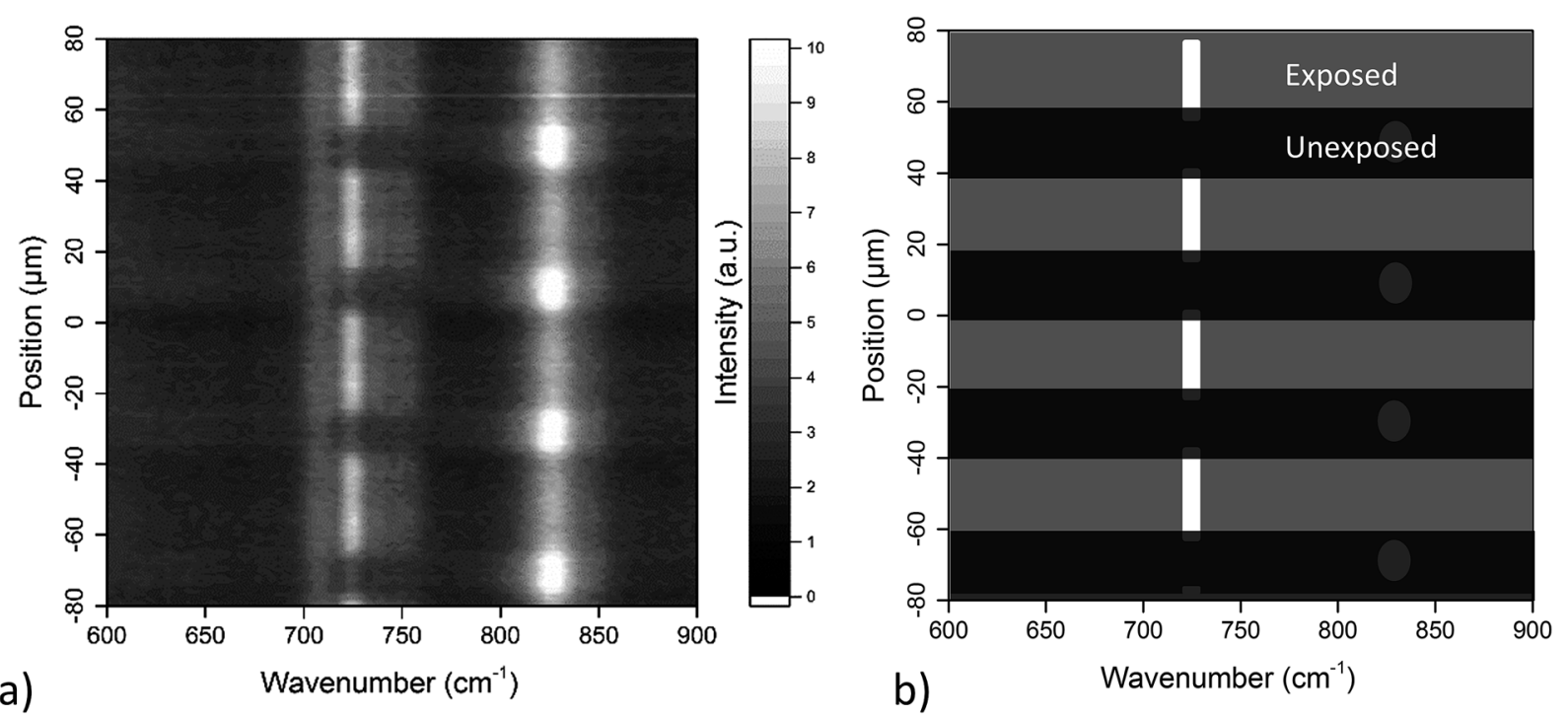

Fig. 6 (a) The Raman spectra of a PFDA-MD40-50 photo-embossed film with respect to position. It can be seen that that $\mathrm{CF}_{3}$ peak (at $725 \mathrm{~cm}^{-1}$ ) of PFDA has a higher intensity in the areas exposed through the photomask. The opposite can be seen for the intensity of the $\mathrm{C}-\mathrm{O}-\mathrm{C}$ peak (at $825 \mathrm{~cm}^{-1}$ ) of the MD40, which has a clear intensity peak in the unexposed areas. This indicates that the PFDA diffuses to the exposed areas. (b) Schematic of graph A where the areas exposed through the photomask and unexposed areas are overlaid. 
the composition varies along the length of the sample (perpendicular to the photomask lines) and that the composition is constant throughout the thickness of the film.

The findings obtained by the confocal Raman measurements strengthens the understanding the photo-embossing model as proposed by Leewis et al. ${ }^{18,19}$ The model is based upon thermodynamic interactions within monomer-monomer and monomer-polymer solutions, showing the influences of monomer reactivity, concentration gradients, cross-linking ability, size, shape and monomer-polymer interaction effects for different mono- and di(meth)acrylate monomer systems. ${ }^{18,19}$ The key differences in this monomeric system are: molecular weight and (number of) reactive groups. The Fomblin MD40 has a molecular weight of $\pm 4200 \mathrm{~g} \mathrm{~mol}^{-1}$ and has two methacrylate groups and the PFDA has a molecular weight of $518 \mathrm{~g} \mathrm{~mol}^{-1}$ and has one acrylate group. Since the Fomblin MD40 has more reactive groups compared to the PFDA it has a higher chance of polymerizing and could be expected to diffuse to the illuminated areas more quickly than the PFDA. However, the reactivity of the methacrylate groups is lower when compared with the acrylate group of the PFDA and the molecular weight of the MD40 is over four times higher than that of PFDA. This results in a faster diffusion of the PFDA compared to the MD40, which has been confirmed by confocal Raman spectroscopy. This is comparable by the polymerization induced diffusion process shown by Liu et al. ${ }^{28}$ Liu et al. showed that the size of the crosslinker used in a polymerization induced diffusion process is key in determining which molecule diffuses to the illuminated areas during polymerization. ${ }^{28}$

To demonstrate pattern fidelity, and the versatility of structures that can be produced using this method, photo-embossed films were also characterised with the scanning electron microscope. Fig. 7 shows several examples of different patterns that can be made by the technique we have described.

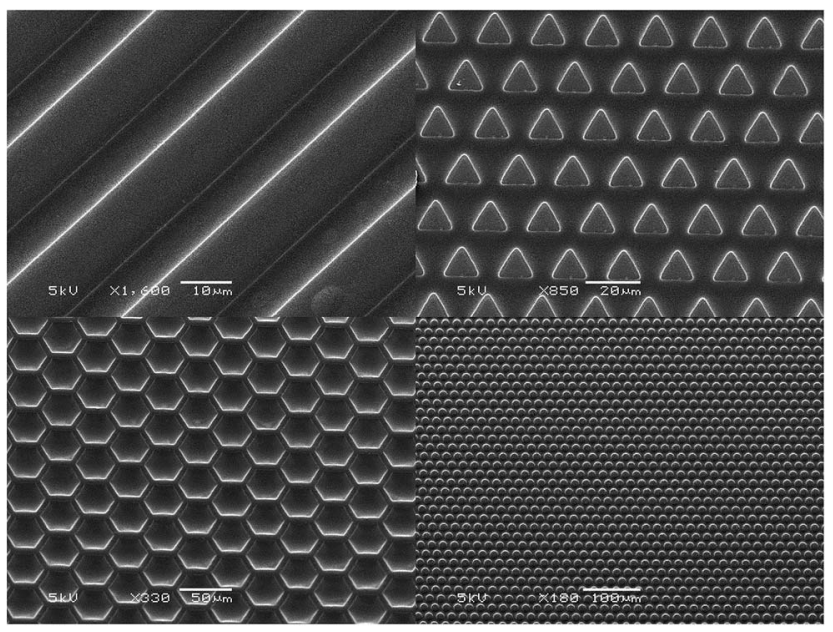

Fig. 7 Example scanning electron micrographs demonstrating the versatility of the surface relief structures that can be generated in the fluoropolymer materials including: lines, triangles, hexagons and circles. All samples are made with a PFDA-MD40-50 monomer mixture with 13 vol\% of DCM. Energy dose was $640 \mathrm{~mJ} \mathrm{~cm}^{-2}$ for mask and flood exposure and the height of the surface structures ranged from 1-3 $\mu \mathrm{m}$ depending on the shape of the feature.
The technique for generating surface relief structures described here has the potential to be scaled up for application on larger surfaces. The flexibility and simplicity of the technique, combined with the chemical simplicity of the fluoropolymer blends, as shown by Yao et al., mean that this system could be combined in a range of application environments. ${ }^{11}$ For example, large area mould application techniques shown by Stenzel, et al. could be easily modified to be compatible with this technique. ${ }^{29,30}$ The adjustments that would be necessary for compatibility with this technique could include changing the flexible mould to a flexible photomask and adding an UV lamp underneath the foil substrate. The low surface energy of the fluoropolymer would ease removal of the film from the flexible photomask. There are however limitations to the photoembossing method, including a limited range for aspect ratios and surface feature heights. However, particularly for lower aspect ratio surface structures, the photo-embossing method is versatile and the height of the surface structures can be changed by adjusting the energy dose and/or intensity without the need for replacing the photomask.

\subsection{Conclusions}

We have demonstrated that surface relief with a range of heights and shapes can be reproducibly formed in fluorinated (meth)acrylates using a modified photo-embossing methodology. Furthermore, the heights of the formed surface relief structures can be controlled by adjusting energy dose, monomer composition and the volume of solvent added to the mixture. Confocal Raman spectroscopy of the resultant films has shown that the concentration of PFDA is higher in the areas exposed to UV light through a photomask compared to the unexposed areas, suggesting that the mechanism of generating these surface relief structures relies on the mass transport of the PFDA towards the exposed areas during photomask exposure. This is expected from the photo-embossing model described by Leewis et al., but this is the first time this has been confirmed with chemical mapping by Raman spectroscopy. The described techniques, in combination with the versatility of the fluoropolymer composition, can potentially result in the design of novel materials incorporating surface relief. Therefore, this simple and versatile technique provides the opportunity to expand fluoropolymer applications.

\section{Acknowledgements}

The authors would like to gratefully acknowledge the financial support of the EU-FP7-SEAFRONT project (614034). The authors would particularly like to acknowledge Dr J. Lavèn for his help with Raman measurements, and T. Bus and K. T. J. Mattheeuwse for their valuable contributions at various stages.

\section{References}

1 A. Vitale, R. Bongiovanni and B. Ameduri, Chem. Rev., 2015, 115, 8835-8866.

2 D. Howell and B. Behrends, Biofouling, 2006, 22, 401-410. 
3 R. J. Crawford, H. K. Webb, V. K. Truong, J. Hasan and E. P. Ivanova, Adv. Colloid Interface Sci., 2012, 179-182, 142-149.

4 J. Yong Chae and B. Bharat, J. Phys.: Condens. Matter, 2010, 22, 035104.

5 R. J. Emerson, T. S. Bergstrom, Y. Liu, E. R. Soto, C. A. Brown, W. G. McGimpsey and T. A. Camesano, Langmuir, 2006, 22, 11311-11321.

6 L. R. J. Scarratt, B. S. Hoatson, E. S. Wood, B. S. Hawkett and C. Neto, ACS Appl. Mater. Interfaces, 2016, 8, 6743-6750.

7 W. Jiang, C. M. Grozea, Z. Shi and G. Liu, ACS Appl. Mater. Interfaces, 2014, 6, 2629-2638.

8 M. Graham and N. Cady, Coatings, 2014, 4, 37-59.

9 K. A. Whitehead, J. Colligon and J. Verran, Colloids Surf., B, 2005, 41, 129-138.

10 C. Christophis, M. Grunze and A. Rosenhahn, Phys. Chem. Chem. Phys., 2010, 12, 4498-4504.

11 X. Yao, S. S. Dunn, P. Kim, M. Duffy, J. Alvarenga and J. Aizenberg, Angew. Chem., 2014, 53, 4418-4422.

12 C. S. Gudipati, J. A. Finlay, J. A. Callow, M. E. Callow and K. L. Wooley, Langmuir, 2005, 21, 3044-3053.

13 T. S. Wong, S. H. Kang, S. K. Tang, E. J. Smythe, B. D. Hatton, A. Grinthal and J. Aizenberg, Nature, 2011, 477, 443-447.

14 M. E. Callow and R. L. Fletcher, Int. Biodeterior. Biodegrad., 1994, 34, 333-348.

15 A. Vitale, M. Quaglio, M. Cocuzza, C. F. Pirri and R. Bongiovanni, Eur. Polym. J., 2012, 48, 1118-1126.

16 E. Kim, Y. Xia and G. M. Whitesides, J. Am. Chem. Soc., 1996, 118, 5722-5731.

17 M. Palacios-Cuesta, I. Vasiev, N. Gadegaard, J. RodríguezHernández and O. García, Eur. Polym. J., 2014, 59, 333-340. 18 C. M. Leewis, A. M. de Jong, L. J. van IJzendoorn and D. J. Broer, J. Appl. Phys., 2004, 95, 4125-4139.
19 C. M. Leewis, A. M. de Jong, L. J. van IJzendoorn and D. J. Broer, J. Appl. Phys., 2004, 95, 8352-8356.

20 C. Sánchez, B. J. de Gans, D. Kozodaev, A. Alexeev, M. J. Escuti, C. van Heesch, T. Bel, U. S. Schubert, C. W. M. Bastiaansen and D. J. Broer, Adv. Mater., 2005, 17, 2567-2571.

21 N. Adams, B.-J. De Gans, D. Kozodaev, C. Sánchez, C. W. M. Bastiaansen, D. J. Broer and U. S. Schubert, J. Comb. Chem., 2006, 8, 184-191.

22 K. Hermans, F. K. Wolf, J. Perelaer, R. A. J. Janssen, U. S. Schubert, C. W. M. Bastiaansen and D. J. Broer, Appl. Phys. Lett., 2007, 91, 174103.

23 J. Perelaer, K. Hermans, C. W. M. Bastiaansen, D. J. Broer and U. S. Schubert, Adv. Mater., 2008, 20, 3117-3121.

24 J. Perelaer, K. Hermans, C. W. M. Bastiaansen, D. J. Broer and U. Schubert, J. Photopolym. Sci. Technol., 2009, 22, 667-670.

25 N. F. Hughes-Brittain, O. T. Picot, M. Dai, T. Peijs and C. W. M. Bastiaansen, Appl. Surf. Sci., 2012, 258, 8609-8612.

26 N. F. Hughes-Brittain, L. Qiu, W. Wang, T. Peijs and C. W. Bastiaansen, J. Biomed. Mater. Res., Part B, 2013, 102, 214-220.

27 O. T. Picot, R. Alcalá, C. Sánchez, M. Dai, N. F. HughesBrittain, D. J. Broer, T. Peijs and C. W. M. Bastiaansen, Macromol. Mater. Eng., 2013, 298, 33-37.

28 D. Liu, C. W. Bastiaansen, J. M. den Toonder and D. J. Broer, Langmuir, 2013, 29, 5622-5629.

29 V. Stenzel, M. Kaune, S. B. C. M. R. Da, Tool for the production of a microstructured surface, WO2005030472 A1, 2005.

30 V. Stenzel, Y. Wilke and W. Hage, Prog. Org. Coat., 2011, 70, 224-229. 